\title{
The Heart Recording Conditions Impact the Assessment of the Electrocardiography Imaging Inverse Solution
}

\author{
Amel Karoui ${ }^{1,2,3}$, Laura Bear ${ }^{1}$, Pauline Migerditichan ${ }^{1,2}$, Mostafa Bendahmane ${ }^{1,2,3}$, Nejib \\ Zemzemi ${ }^{1,2,3}$ \\ ${ }^{1}$ Electrophysiology and Heart Modeling Institute (IHU-Lyric), Bordeaux, France \\ ${ }^{2}$ French Institute for Research in Computer Science and Automation (INRIA), Bordeaux, France \\ ${ }^{3}$ Institute of Mathematics, University of Bordeaux, France
}

\begin{abstract}
Our purpose is to assess different methods for choosing the regularization parameter combined with the Method of Fundamental Solutions (MFS) for solving the electrocardiography imaging (ECGi) inverse problem but also to assess their sensitivity to the nature of recorded signals. Results are provided using left, right and bi-ventricular pacing experiments performed in an exvivo pig heart. In the experiment, some electrodes are too close to the pericardium so that they seem to be measuring monophasic action potential signals (MAPs) rather then extracellular epicardial potential. The electrodes suspected to measure MAPs are identified by thresholding the epicardial potential in the plateau phase. This leads to compare the computed epicardial potential to measurement results with and without considering these electrodes measurements. Removing the MAPs electrodes improves the quality of the RE and CC by at least $5 \%$. Results show that the Generalized Cross Validation (GCV) approach provides the best results in the three pacing cases.
\end{abstract}

\section{Introduction}

The electrocardiographic imaging (ECGi) is a noninvasive technique providing the electrical potential on the epicardial surface from measures realized on the thoracic surface using a set of electrodes. It's based on a mathematical model which describes the heart-torso electrical activity. This is called an inverse problem and is known to be ill-posed resulting from different factors such as the uncertainty of the mathematical model. In fact, modeling the electrical activity by a laplacian equation and considering the heart-torso domain as homogeneous generate modeling errors. In addition, experimental protocols yields measurement errors and geometries' innaccuracy especially in determining the heart position $[1,2]$. These conditions can generate large and even discontinuous errors in the inverse solution. Regularization procedures are then used to stabilize the solution and ensure its uniqueness and its dependence on the data. To date, many regularization approaches are suggested. In this work, we depicted the zeroorder Tikhonov regularization, presumed to be the most simple and appropriate non-a-priori regularization method to solve the inverse problem of electrocardiography [3]. One of the issues of the regularization methods is the choice of the regularization parameter. Actually, few works have compared the different developed approaches [4-6]. In this study, we compare different regularization parameter choice approaches combined with the Method of Fundamental Solutions using an experimental set of data containing monophasic action potential-like signals presumed to be ischemic ones in order to assess their effects on the reconstruction process.

\section{Methods}

The regularization approach most commonly used to solve the ECGi inverse problem is the Tikhonov regularization defined by the following objective function:

$$
\min _{x}\left\{\|A x-b\|^{2}+\lambda^{2}\|L x\|^{2}\right\},
$$

where $\mathbf{A}$ is the transfer matrix defined by MFS, $\mathbf{b}$ is the vector illustrating the Dirichlet and Neumann boundary conditions and $\mathbf{x}$ is a vector of weighting coefficients used to compute the epicardial potential in any point of the torso domain. The regularization operator $\mathbf{L}$ can be the identity matrix for zero-order Tikhonov or a gradient operator for first or second order regularization. The $\lambda$ is the regularization parameter and $\|$.$\| is the L2-norm. Here,$ $\mathbf{L}$ is the identity matrix.

To date, the use of the Singular Value Decomposition (SVD) of $\mathbf{A}$ is the best way to assess the different regularization parameter choice methods. Following [7], 
we decompose $A$ as follows :

$$
A=U \Sigma V^{T}=\sum_{i=1}^{n} u_{i} \sigma_{i} v_{i}^{T},
$$

where $\mathbf{U}$ is a $m \times n$ orthonormal matrix containing the left singular vectors of $\mathbf{A}, \mathbf{V}$ is a $n \times n$ orthonormal matrix containing the right singular vectors of $\mathbf{A}$ and $\Sigma$ is a $n \times n$ diagonal matrix with the singular values of $\mathbf{A}$ on its diagonal. Note that $u_{i}, v_{i}$ and $\sigma_{i}$ are respectively, the columns of $\mathbf{U}, \mathbf{V}$ and the singular values of $\mathbf{A}$ arranged in a decreasing order. The solution of the regularized problem is expressed, using the SVD, by:

$$
x=A^{*} b=\left(A^{T} A+\lambda^{2} I\right)^{-1} A^{T} b=\sum_{i=1}^{n} \frac{\sigma_{i}^{2}}{\sigma_{i}^{2}+\lambda^{2}} \frac{u_{i}^{T} b}{\sigma_{i}} v_{i} .
$$

As shown in [8], the two terms of (1) can be written as :

$$
\rho(\lambda)=\|A x-b\|^{2}=\sum_{i=1}^{n} \frac{\lambda^{4} \mu_{i}^{2}}{\left(\lambda^{2}+\sigma_{i}^{2}\right)^{2}}+\left\|r_{\perp}\right\|^{2},
$$

and

$$
\eta(\lambda)=\|x\|^{2}=\sum_{i=1}^{n} \frac{\sigma_{i}^{2} \mu_{i}^{2}}{\left(\lambda^{2}+\sigma_{i}^{2}\right)^{2}},
$$

where $\left\|r_{\perp}\right\|^{2}=\left\|A x_{L S S}-b\right\|^{2}$ is the residual of the least squares solution $x_{L S S}$ and $\mu_{i}=u_{i}^{T} b$.

\subsection{Determination of the Regularization Parameter}

\subsubsection{U-Curve}

The U-Curve is a plot of the sum of the inverse of $\eta(\lambda)$ and the inverse of the corresponding residual $\rho(\lambda)$ in terms of $\lambda$ on a $\log -\log$ scale:

$$
\operatorname{Ucurve}(\lambda)=\frac{1}{\rho(\lambda)}+\frac{1}{\eta(\lambda)} .
$$

The U-Curve method was first proposed by [9]. It has been shown that $U$ curve $(\lambda)$ reaches a local minimum $\lambda_{u}$, the optimum value of $\lambda$, in the interval $\left[\delta_{n}^{2 / 3}, \delta_{1}^{2 / 3}\right]$, where $\delta_{1}$ and $\delta_{n}$ are respectively the biggest and the smallest singular values.

\subsubsection{ADPC}

ADPC is a regularization parameter choice method based on the Discrete Picard Condition (DPC) [10]. The idea is to look for the last index $i$ before the DPC is no longer satisfied [11]. This means before $\sigma_{i}$ becomes smaller than $\left|u_{i}^{T} b_{t}\right|$ in a $\log$-log scale where $t$ is time.
For the sake of simplification, $\log \left(\left|u_{i}^{T} b_{t}\right|\right)$ is fitted by a polynomial $p_{t}\left(i, \log \left(\left|u_{i}^{T} b_{t}\right|\right)\right)$ of degree 5 to 7 . Then, for each $p_{t}$, we seek for $\alpha_{t}=\sigma_{j}$ such that $\log \left(\sigma_{i}\right) \geq p_{t}$, for all $i<j$. The ADPC regularization parameter is then $\lambda=\operatorname{median}\left(\alpha_{t}\right)$.

\subsubsection{CRESO}

According to the Composite REsidual and Smoothing Operator (CRESO) method [12], the optimal $\lambda$ corresponds to the first local maximum of the derivative of the difference between the constraint term and the residual term with respect to $\lambda^{2}$.

$$
C(\lambda)=\frac{d}{d\left(\lambda^{2}\right)}\left(\lambda^{2} \eta(\lambda)-\rho(\lambda)\right) .
$$

In terms of SVD, this can be written as [8]:

$$
C(\lambda)=\sum_{i=1}^{n} \frac{\sigma_{i}^{2} \mu_{i}^{2}\left(\sigma_{i}^{2}-3 \lambda^{2}\right)}{\left(\sigma_{i}^{2}+\lambda^{2}\right)^{3}}
$$

\subsubsection{GCV}

The idea behind The Generalized-Cross Validation (GCV) [13] is that the optimum of the regularization parameter provides the best prediction of a measurement as a function of the others. It provides the optimal value of $\lambda$ by minimizing the function :

$$
G(\lambda)=\frac{\rho(\lambda)}{\left[\operatorname{Trace}\left(I-A A^{*}\right)\right]^{2}} .
$$

In terms of SVD, $G(\lambda)$ is expressed by :

$$
G(\lambda)=\frac{\sum_{i=1}^{n} \frac{\lambda^{4} \mu_{i}^{2}}{\left(\sigma_{i}^{2}+\lambda^{2}\right)^{2}}+\left\|r_{\perp}\right\|^{2}}{\left(m-\sum_{i=1}^{n} \frac{\sigma_{i}^{2}}{\sigma_{i}^{2}+\lambda^{2}}\right)^{2}} .
$$

\subsubsection{RGCV}

In [14], the RGCV estimate is defined by the minimizer of the following function:

$$
R(\lambda)=[\gamma+(1-\gamma) \xi(\lambda)] G(\lambda),
$$

where $G(\lambda)$ is given by (9) and $\xi(\lambda)$ is defined as:

$$
\xi(\lambda)=\text { Trace }\left[\left(A A^{*}\right)^{2}\right]=\sum_{i=1}^{n} \frac{\sigma_{i}^{4}}{\left(\lambda^{2}+\sigma_{i}^{2}\right)^{2}} .
$$

Here, $\gamma$ is called a robustness parameter, $\gamma \in[0,1]$. The RGCV method is based on the average influence $\frac{1}{m} \sum_{i=1}^{m}\left\|A x_{\lambda}-A x_{\lambda}^{[i]}\right\|^{2}$, where $\left\|A x_{\lambda}-A x_{\lambda}^{[i]}\right\|^{2}$ is a measure of the influence of the $i^{t h}$ data point on the regularized solution. 


\subsection{Ex-vivo experimental data and metrics}

Experimental data were obtained using an ex-vivo pig heart perfused in Langendorff mode suspended into a human-shaped torso tank paced on the left and right ventricular surfaces. Epicardial ventricular electrograms were recorded using a 108-electrode sock, from which 93 were used, simultaneously with torso potentials from 128 electrodes embedded in the tank surface as it appears in Figure 1. The tank mesh contains 1234 nodes and the epicardium 649 nodes. More details about the ex-vivo experimental protocol can be found in Bear et al [15].

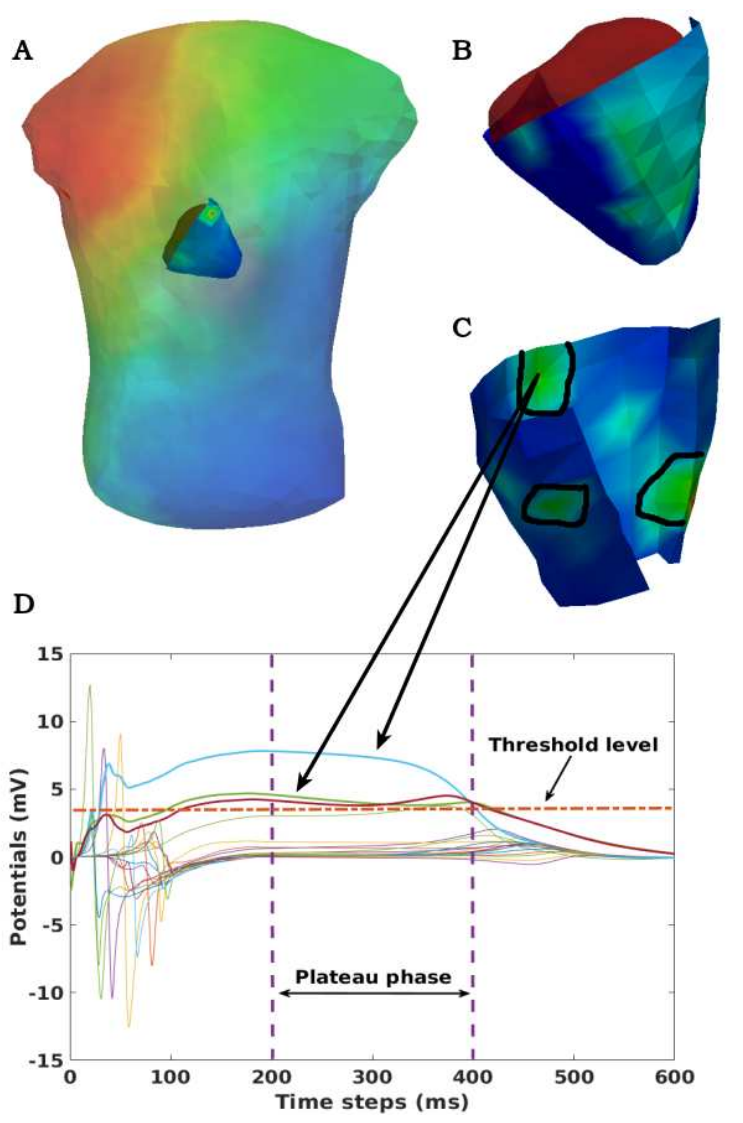

Figure 1. A : The heart-human shaped torso tank model used for the experiments, B : The segmented pig heart covered by the sock used for potential records, C : Measured Epicardial potential map at a sample time of the plateau phase. The surrounded zones are ischemic regions, D : Measured MAP-like electrograms.

Preprocessing of the experimental data revealed the existence of a few localized sites of ischemia produced due to electrode pressure on the epicardium represented in Figure 1C. This produced monophasic action potentiallike signals (MAPs). These electrodes are identified when the potential is greater than a fixed threshold equal to $50 \%$ of the maximum signal magnitude in the plateau phase, $250 \mathrm{~ms}$ after pacing as it's illustrated in Figure 1D. This choice is based on observations of the QT interval in order to eliminate the ischemic signals. This leads us to run two sets of comparisons, with all the working electrodes (WE) and after removing the above threshold electrodes (TE). According to the ex-vivo experimental protocol, the sock doesn't cover the whole surface of the epicardium. So, a linear interpolation was applied to the ex-vivo recordings with and without thresholding to obtain respectively potential recordings (TE) and (WE) for all the mesh nodes.

To assess the performance of the different methods, we use the standard metrics : the relative error (RE) and the correlation coefficient (CC).

\section{Results}

Table 1 presents the mean RE and $\mathrm{CC}$ of the reconstructed epicardial potentials using the different regularization parameter choice methods combined with MFS for the 3 experiences : RV, LV and BiV. Also, we compare, in this table, the results obtained using the nonthresholded signals (WE) versus those computed with the thresholded signals (TE). Results show that thresholding improves the RE from 0.92 to 0.80 for RV, from 0.78 to 0.72 for $\mathrm{LV}$ and from 0.99 to 0.72 for $\mathrm{BiV}$, using GCV. CC values are also improved. Regarding the regularization parameter choice methods, we observe that GCV outperforms the other methods.

\begin{tabular}{cccccccc} 
& & \multicolumn{9}{c}{ Simulation } \\
\cline { 2 - 8 } & & \multicolumn{2}{c}{ RVP } & \multicolumn{2}{c}{ LVP } & \multicolumn{2}{c}{ BiVP } \\
\hline \hline Method & Data set & RE & CC & RE & CC & RE & CC \\
\hline \multirow{2}{*}{ CRESO } & WE & 0.93 & 0.32 & 0.84 & 0.61 & 1.00 & 0.40 \\
\cline { 2 - 8 } & TE & 0.82 & 0.57 & 0.81 & 0.66 & 0.81 & 0.66 \\
\hline \hline \multirow{2}{*}{ GCV } & WE & 0.92 & 0.31 & 0.78 & 0.67 & 0.99 & 0.34 \\
\cline { 2 - 8 } & TE & 0.80 & 0.60 & 0.72 & 0.72 & 0.72 & 0.72 \\
\hline \multirow{2}{*}{ RGCV } & WE & 0.93 & 0.32 & 0.86 & 0.61 & 1.00 & 0.40 \\
\cline { 2 - 8 } & TE & 0.85 & 0.54 & 0.84 & 0.66 & 0.84 & 0.66 \\
\hline \hline \multirow{2}{*}{ U-Curve } & WE & 0.94 & 0.33 & 0.98 & 0.6 & 1.02 & 0.40 \\
& TE & 0.96 & 0.53 & 0.97 & 0.65 & 0.97 & 0.65 \\
\hline \multirow{2}{*}{ ADPC } & WE & 0.92 & 0.31 & 0.82 & 0.62 & 0.98 & 0.38 \\
\cline { 2 - 8 } & TE & 0.82 & 0.56 & 0.78 & 0.67 & 0.78 & 0.67 \\
\hline
\end{tabular}

Table 1. Means of RE and CC of the reconstructed epicardial potentials using MFS combined with the different regularization parameter choice methods for thresholded data sets and non-thresholded ones.

Figure 2 shows a measured monophasic action potential-like signal and the corresponding thresholded one with the reconstructed version by MFS-GCV. We observe that the thresholding affects the results essentially during the plateau phase where it reduces the gap between the reconstructed and the measured amplitudes. 

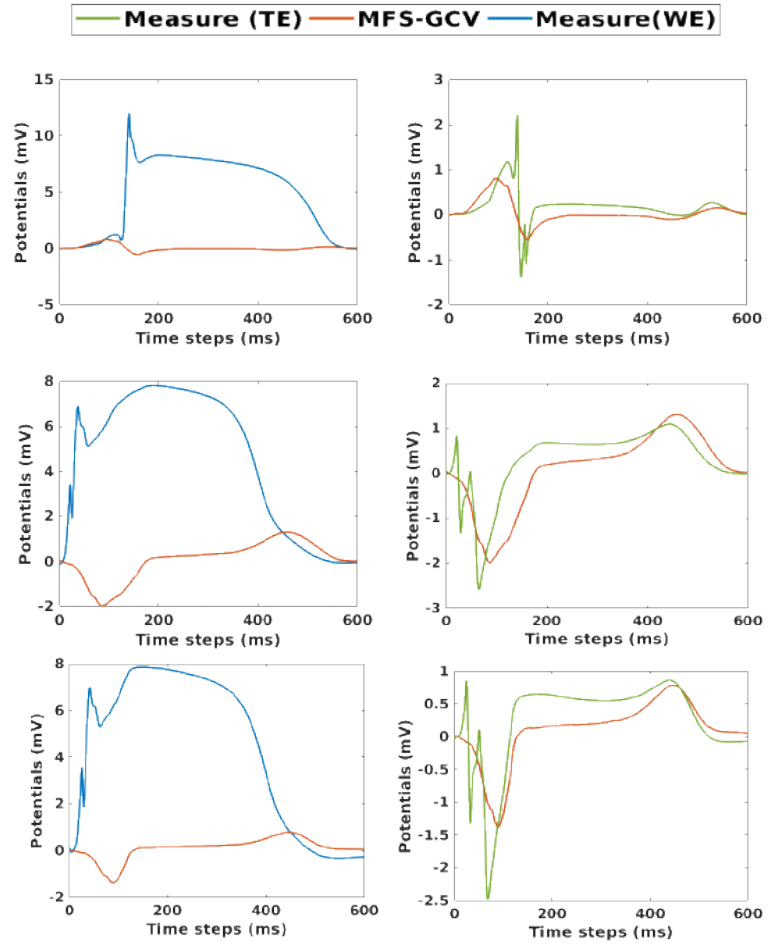

Figure 2. Measured (WE), thresholded (TE) and reconstructed electrograms of the RV (A), LV (B) and $\mathrm{BiV}$ (C) in the same ischemic-like point using MFS-GCV.

\section{Discussion and conclusion}

In this paper, we assessed 5 methods for choosing the regularization parameter for a zero-order Tikhonov regularization combined with MFS : CRESO, GCV, RGCV, U-Curve and ADPC. Results show that all the methods work well combined with MFS. However, GCV outperforms all the other methods in terms of RE and CC for the three cases : RV, LV and BiV.

Regarding the thresholding applied to the reference potential measures, results seem to be better using the thresholded epicardial potentials. Nevertheless, we can't decide on the impact of the monophasic action potentiallike signals on the quality of the results unless we have experiments providing non ischemic potentials.

\section{References}

[1] Messinger-Rapport BJ, Rudy Y. Computational issues of importance to the inverse recovery of epicardial potentials in a realistic heart-torso geometry. Mathematical Biosciences 1989;97(1):85-120.

[2] Messinger-Rapport BJ, Rudy Y. Regularization of the inverse problem in electrocardiography: A model study. Mathematical Biosciences 1988;89(1):79 - 118. ISSN 0025-5564.

[3] Figuera C, Suárez-Gutiérrez V, Hernández-Romero I,
Rodrigo M, Liberos A, Atienza F, Guillem MS, BarqueroPérez Ó, Climent AM, Alonso-Atienza F. Regularization techniques for ecg imaging during atrial fibrillation: a computational study. Frontiers in physiology 2016;7:466.

[4] Cheng LK, Bodley JM, Pullan AJ. Comparison of potentialand activation-based formulations for the inverse problem of electrocardiology. IEEE Transactions on Biomedical Engineering 2003;50(1):11-22.

[5] Barnes JP, Johnston PR. Application of robust generalised cross-validation to the inverse problem of electrocardiology. Computers in biology and medicine 2016;69:213-225.

[6] Bauer F, Lukas MA. Comparingparameter choice methods for regularization of ill-posed problems. Mathematics and Computers in Simulation 2011;81(9):1795-1841.

[7] Hansen PC. Rank-Deficient and Discrete Ill-posed Problems. Philadelphia: SIAM, 1998.

[8] Johnston P, Gulrajani R. A new method for regularization parameter determination in the inverse problem of electrocardiography. IEEE Transactions on Biomedical Engineering 1997;44(1):19-39.

[9] Krawczyk-Stado D, Rudnicki M. Regularization parameter selection in discrete ill-posed problems - the use of the ucurve. International Journal of Applied Mathematics and Computer Science 2007;17(2):157-164.

[10] Hansen PC. Truncated singular value decomposition solutions to discrete ill-posed problems with ill-determined numerical rank. SIAM Journal on Scientific and Statistical Computing 1990;11(3):503-518.

[11] Chamorro-Servent J, Dubois R, Potse M, Coudière Y. Improving the spatial solution of electrocardiographic imaging: A new regularization parameter choice technique for the tikhonov method. In Pop M, Wright GA (eds.), Functional Imaging and Modelling of the Heart. Cham: Springer International Publishing. ISBN 978-3-319-594484, 2017; 289-300.

[12] Colli-Franzone P, Guerri L, Tentoni S, Viganotti C, Baruffi S, Spaggiari S, Taccardi B. A mathematical procedure for solving the inverse potential problem of electrocardiography. analysis of the time-space accuracy from in vitro experimental data. Mathematical Biosciences 1985;77(1):353 - 396. ISSN 0025-5564.

[13] Wahba G. Practical approximate solutions to linear operator equations when the data are noisy. SIAM Journal on Numerical Analysis 1977;14(4):651-667.

[14] Lukas MA. Robust generalized cross-validation for choosing the regularization parameter. Inverse Problems 2006;22(5):1883.

[15] Bear LR, Huntjens PR, Walton R, Bernus O, Coronel R, Dubois R. Cardiac electrical dyssynchrony is accurately detected by noninvasive electrocardiographic imaging. Heart Rhythm 2018;ISSN 1547-5271.

Address for correspondence:

Amel Karoui

Team Carmen, INRIA Bordeaux sud-ouest

200 rue de la Vieille Tour, 33405 Talence, France

amel.karoui@inria.fr 